\title{
Influência da área da parcela e do número de repetições na precisão de experimentos com arbóreas ${ }^{(1)}$
}

\begin{abstract}
Adroaldo Guimarães Rossetti(2)
Resumo - Os experimentos de campo com plantas perenes arbóreas ocupam grandes áreas, em razão do porte das plantas que exigem largos espaçamentos. É freqüente usarem-se parcelas grandes, em detrimento do número de repetições, para diminuir a área experimental, a mão-de-obra e o conseqüente custo da pesquisa. Essa prática, contudo, traz prejuízos à precisão das estimativas dos parâmetros e à aplicação eficiente de testes estatísticos. Este trabalho teve o objetivo de mostrar que o uso de parcelas pequenas favorece o aumento do número de repetições, diminui a área dos experimentos e aumenta a sua precisão. $\mathrm{O}$ método utilizado associa o tamanho da unidade experimental ao número de repetições, pela minimização da variância da média de cada tratamento, permitindo maior número de repetições, para aumentar a precisão dos testes, obter maior uniformidade no experimento e melhorar a qualidade das pesquisas. Parcelas pequenas favorecem o aumento do número de repetições, permitem obter melhores estimativas do erro experimental, dos efeitos de tratamentos e dos parâmetros, dando mais eficiência aos testes estatísticos aplicados aos dados. Houve diminuição do número de plantas necessárias ao experimento e redução da área experimental em cerca de $18 \%$.
\end{abstract}

Termos para indexação: plantas perenes, experimentação, métodos, análise estatística.

\section{Influence of the plot size and number of replicates on the precision of perennial arboreal plant trials}

\begin{abstract}
Field experiments with perennial arboreal trees usually occupy large areas due to the big plant size and the required spacings. In such situations the use of big plots and the low number of replicates are very common, in an attempt to reduce the experimental area, manpower and costs. This procedure, however, decreases the efficiency of results and difficults the application of statistical tests. This work was conducted in order to demonstrate that using small plots and higher number of replicates increases the trial precision and decreases the experimental area. The association of plot size and number of replicates, minimizing the mean variance of each treatment, allows the use of high number of replicates, increasing the precision of statistical tests, besides a better trial uniformity. The use of smaller plots benefits the increase of replicates number, allows a better estimation of the experimental error, of the treatment and parameters effects and a higher efficiency of statistical tests. Besides, it reduces about $18 \%$ the number of plants and the size of experimental area.
\end{abstract}

Index terms: perennial plants, experimentation, methods, statistical analysis.

\section{Introdução}

A literatura sobre métodos e técnicas experimentais para pesquisas com plantas perenes e frutíferas arbóreas, sobretudo em relação a experimentos de campo, é bastante escassa. Pearce (1975), para tentar preencher essa lacuna, propôs métodos para ex-

\footnotetext{
(1) Aceito para publicação em 21 de maio de 2001.

(2) Embrapa-Centro Nacional de Pesquisa de Agroindústria Tropical, Caixa Postal 3761, CEP 60511-110 Fortaleza, CE E-mail: adroaldo@cnpat.embrapa.br
}

perimentos com frutíferas e outras plantas perenes. Apesar de um bom indicativo, não se trata de resultados de pesquisa, mas de inferências feitas sobre resultados obtidos com cultivos de ciclo curto, que normalmente não se adaptam bem às perenes arbóreas, cujos experimentos de campo ocupam, em geral, grandes áreas, em virtude dos largos espaçamentos exigidos, o que tem causado, segundo Rossetti (1994), alguns problemas, com reflexos nos resultados das pesquisas. Não é rara a existência de experimentos com parcelas grandes, com poucas ou sem repetições, sob a alegação de obter maior stand e maior facilidade de manejo. É comum, todavia, perguntar-se qual o número mínimo de repetições que 
devem ter os tratamentos de um experimento de campo, com esses cultivos, justamente pelo aumento da área experimental e suas conseqüências, para obtenção de resultados satisfatórios. A repetição, que constitui um dos princípios básicos da experimentação científica, objetiva propiciar estimativas do erro experimental, tão importante nos testes dos efeitos dos tratamentos e dos parâmetros dos modelos usados nas pesquisas realizadas por experimentos, e permitir a distribuição dos tratamentos em maior espaço do ambiente, com vistas a adequar a extrapolação dos resultados obtidos. Dagnelie (1977) demonstrou que quanto maior for o número de repetições, mais fidedigna será a estimativa do erro experimental, portanto, haverá mais segurança na aplicação dos testes estatísticos e maior confiabilidade nos resultados. Por outro lado, dependendo do número de tratamentos, do tamanho da parcela e do delineamento experimental utilizado, o experimento pode crescer tanto que às vezes se torna impraticável. É exatamente por isso que muitos experimentos utilizam parcelas grandes, com grande número de plantas, em detrimento do número de repetições, o que é bastante grave quanto à estimativa do erro experimental e, em última análise, aos resultados obtidos nesses experimentos, uma vez que, em geral, as parcelas grandes têm maior variância (Rossetti et al., 1996), e conseqüentemente aumentam a variância da média de cada tratamento.

A determinação prévia do número mínimo de repetições necessárias para se obter o melhor resultado tem sido bastante discutida, com muitas soluções propostas, mas, segundo Pimentel-Gomes (1990), nenhuma é inteiramente satisfatória. Por sua vez, o uso de parcelas de tamanho ótimo contribui para que se ajuste um razoável número de repetições para obtenção de resultados satisfatórios. Essas parcelas, segundo Rossetti \& Pimentel-Gomes (1983), em geral associadas a delineamentos ou técnicas experimentais apropriados, como blocos incompletos ou fatoriais fracionários, por exemplo, conforme o objetivo da pesquisa, como recomendam vários autores, entre eles Rossetti (1994), contribuem bastante para minimizar a variabilidade normalmente existente nesses cultivos. Tais práticas, entretanto, exigem, quase sempre, genótipos bem definidos em estudos de melhoramento. Os experimentos iniciais dessa área, em que a interação genótipo $v s$. ambiente tem particular importância, e de outras, normalmente com grande número de tratamentos, têm sido os mais atingidos. No outro extremo estão os experimentos com poucos tratamentos, que a literatura preconiza que se faça mais de uma repetição no bloco, no caso dos delineamentos de blocos ao acaso, para que se tenha, conforme Banzatto \& Kronka (1995), maior número de graus de liberdade para estimar o erro experimental. Este é, sem dúvida, um recurso interessante, nesse sentido, mas quanto ao aspecto da distribuição dos tratamentos em maior espaço do ambiente, para estabelecer a validade de extrapolação dos resultados, esta fica prejudicada, pois a área experimental, normalmente pequena, restringe os resultados àquelas condições. Isso indica a necessidade de estudo mais profundo na relação entre tamanho de parcelas e número de repetições. Pearce \& Moore (1976) deram boa contribuição na forma de redução do erro experimental nos experimentos com plantas perenes, considerando a correlação que há entre parcelas vizinhas, independentemente do tamanho, sem contudo relacioná-las com o número de repetições. McRae \& Ryan (1996) mencionam vários aspectos do planejamento de experimentos de longa duração, no contexto de ajuste de modelos e técnicas experimentais, entre os quais o tamanho da parcela e o número de repetições, e outros fatores que normalmente ocorrem ao longo do tempo, sem, contudo, estabelecer relação entre eles. Swallow (1981) mostrou a importância do bom planejamento dos experimentos com plantas perenes, nos quais estabeleceu alguns métodos estatísticos, no contexto de análise dos dados, considerando as medidas feitas ao longo do tempo, nesses experimentos, sem, contudo, tratar dessa relação. Ortiz (1995) estimou tamanho e forma de parcelas para pesquisas com a cultura da bananeira, pelo método da máxima curvatura, que além de variar conforme a escala usada para a projeção da curva, não leva em conta a variabilidade que existe entre parcelas vizinhas. Quanto ao número de repetições, limitou-se a se referir a outros autores. Genezi et al. (1980) estimaram tamanhos ótimos de parcelas para bananeira, pelo método empírico, proposto por Smith (1938), que leva em conta a heterogeneidade do solo, mas não considera a correlação que existe entre parcelas vizinhas nem estabelece nenhuma re- 
lação entre o tamanho da parcela e o número de repetições.

O objetivo deste trabalho foi mostrar que o uso de parcelas pequenas, nos experimentos com plantas perenes arbóreas, favorece o aumento do número de repetições, diminui a área dos experimentos e aumenta a sua precisão.

\section{Material e Métodos}

Considerando-se um experimento com c clones de certa cultura perene, em b blocos casualizados, com k plantas úteis por parcela, em que os dados são obtidos de cada planta, cujo modelo estatístico, segundo Pimentel-Gomes (1984), é escrito como:

$\mathrm{Y}_{\mathrm{ijk}}=\mathrm{m}+\mathrm{c}_{\mathrm{i}}+\mathrm{b}_{\mathrm{j}}+\mathrm{e}_{\mathrm{ijk}}$

onde:

m é a média; $\mathrm{c}_{\mathrm{i}}(\mathrm{i}=1,2, \ldots, \mathrm{c})$ é o efeito do i-ésimo clone; $b_{j}(j=1,2, \ldots, b)$ é o efeito do j-ésimo bloco; $\mathrm{e}_{\mathrm{ijk}}$ são aleatórios, com $\mathrm{E}\left(\mathrm{e}^{2}{ }^{2}{ }^{\mathrm{jk} k}\right)=\sigma^{2}, \mathrm{E}\left(\mathrm{e}_{\mathrm{ijk}} \mathrm{e}_{\mathrm{i}^{\prime}{ }^{\prime} \mathrm{k}^{\prime}}\right)=0$ para $(\mathrm{i}, \mathrm{j}) \neq\left(\mathrm{i}^{\prime}, \mathrm{j}^{\prime}\right)$, $\mathrm{E}\left(\mathrm{e}_{\mathrm{ijk}} \mathrm{e}_{\mathrm{i}^{\prime}{ }^{\prime} \mathrm{k}^{\prime}}\right)=\rho \sigma^{2}$, onde $\rho$ é o coeficiente de correlação intraclasse, de onde decorre a análise de variância apresentada na Tabela 1.

$\operatorname{Com} \rho \geq 0, E\left(V_{1}\right) \geq E\left(V_{2}\right)$, para $k \geq 1$. Mas com $\rho<0$ temse a possibilidade de obter $E\left(V_{1}\right)<E\left(V_{2}\right)$, o que é importante, pois deve-se ter sempre

$\sigma^{2}[1+(k-1) \rho] \geq 0$, ou seja: $\rho \geq-\frac{1}{k-1}$, para $(k>1)$.

Essa expressão indica que, quando aumenta o valor de $k$, o valor de $\rho$, se inicialmente negativo, estará cada vez mais próximo de zero, isto é, para $\rho<0$, tem-se:

$\lim \rho=0 \forall \mathrm{k} \rightarrow \infty$.

Em qualquer caso tem-se sempre: $-1 /(\mathrm{k}-1) \leq \rho \leq 1,(\mathrm{k}>1)$.

Da análise de variância antes exposta, obtém-se um estimador de $\rho$, pela expressão:

$\hat{\rho}=\left(\mathrm{V}_{1}-\mathrm{V}_{2}\right) /\left[\mathrm{V}_{1}+(\mathrm{k}-1) \mathrm{V}_{2}\right],(\mathrm{k}>1)$.

Conclui-se daí que:

$\mathrm{V}_{1} \geq \mathrm{V}_{2} \Rightarrow \hat{\rho} \geq 0, \mathrm{e}$

$\mathrm{V}_{1}<\mathrm{V}_{2} \Rightarrow \hat{\boldsymbol{\rho}}<0$.

Tabela 1. Componentes da análise de variância de um experimento com c clones de uma cultura perene, com b bloco casualizadas e $\mathrm{k}$ plantas úteis por parcela.

\begin{tabular}{llcc}
\hline Fonte de variação & GL & QM & E(QM) \\
\hline Blocos & $(\mathrm{b}-1)$ & - & - \\
Clones & $(\mathrm{c}-1)$ & - & - \\
Resíduo (a) & $(\mathrm{b}-1)(\mathrm{c}-1)$ & $\mathrm{V}_{1}$ & $\sigma^{2}[1+(\mathrm{k}-1) \rho]$ \\
Resíduo (b) & $\mathrm{bc}(\mathrm{k}-1)$ & $\mathrm{V}_{2}$ & $\sigma^{2}(1-\rho)$ \\
\hline
\end{tabular}

Por outro lado, sendo $V_{1}$ e $V_{2}$ positivos, tem-se necessariamente:

$-1 /(\mathrm{k}-1)<\hat{\rho}<1$, para $\mathrm{k}>1$.

Note-se que, para $0 \leq \hat{\rho}<1$, tem-se:

$\frac{\hat{\rho}}{1-\hat{\rho}}=\left(\frac{\sigma_{2}}{\sigma_{1}}\right)^{2}$.

O cálculo do coeficiente de variação será feito pela variância relativa de cada parcela que tem a expressão: $\mathrm{V}_{1}=\sigma^{2}[1+(\mathrm{k}-1) \hat{\rho}]$.

Conclui-se, portanto, que sendo $\mathrm{m}$ a média geral, o coeficiente de variação relativo ao resíduo (a), referente a parcelas, é obtido pela expressão:

$\mathrm{CV}=(100 / \mathrm{km}) \sqrt{\sigma^{2}[1+(\mathrm{k}-1) \hat{\rho}]}$

$\mathrm{CV}=(100 \sigma / \mathrm{m}) \sqrt{\frac{1-\hat{\rho}}{\mathrm{k}^{2}}+\frac{\hat{\rho}}{\mathrm{k}}}$

$\mathrm{CV}=(100 \sigma / \mathrm{m}) \sqrt{\frac{(1-\hat{\rho})+\mathrm{k} \hat{\rho}}{\mathrm{k}^{2}}}$,

$k>0$, para $1+(k-1) \hat{\rho} \geq 0$.

A média de plantas $\mathrm{m}$ é uma função decrescente de $\mathrm{k}$.

Com $\hat{\rho} \geq 0$, o máximo do $C V$ se dá para $\mathrm{k}=1$, pois ambos os termos do radicando $\left[(1-\hat{\rho}) / k^{2}\right]+(\hat{\rho} / k)$ são funções decrescentes de $\mathrm{k}$. Com $\hat{\rho}=1$, o primeiro termo é nulo, mas isso não altera o resultado.

Com $\hat{\rho}<0$, o numerador do radicando $(1-\hat{\rho})+k \hat{\rho}$ decresce quando $\mathrm{k}$ cresce, mas seu valor é restringido pela condição necessária de que se tenha $(1-\hat{\rho})+\mathrm{k} \hat{\rho} \geq 0$. Ao mesmo tempo, cresce o denominador $\mathrm{k}^{2}$, de modo que o radicando é monotonicamente decrescente, para $\mathrm{k} \geq 1$.

Conclui-se, finalmente, que o coeficiente de variação é função decrescente do número $\mathrm{k}$ de plantas úteis por parcela, o que favoreceria o uso de parcelas grandes, contribuindo para o aumento da variância da média de cada tratamento, dificultando, quando existissem, a detecção de diferenças significativas entre eles. Nesse caso, o mais importante é reduzir a variância da média de cada tratamento, sem aumentar o número de plantas do experimento, isto é, para uma área fixa ou um número fixo de plantas, tornar mínima a variância da média de cada tratamento a fim de que se possa detectar diferenças significativas entre eles, quando estas existirem.

Considerando o modelo expresso anteriormente, a variância da média de $r$ repetições de cada tratamento é obtida pela expressão:

$\mathrm{V}(\hat{\mathrm{m}})=\left(\frac{\sigma^{2}}{\mathrm{kr}}\right)[1+(\mathrm{k}-1) \hat{\rho}]$, para $\mathrm{k}, \mathrm{r}>1$ e $\hat{\rho}>0$.

Não havendo bordadura entre as parcelas e sendo $\mathrm{N} \mathrm{o}$ número total de plantas por tratamento, tem-se $\mathrm{N}=\mathrm{kr}$, logo: 
$\mathrm{V}(\hat{\mathrm{m}})=\left(\frac{\sigma^{2}}{\mathrm{~N}}\right)[1+(\mathrm{k}-1) \hat{\rho}]$.

Com $\hat{\rho}>0$, essa variância é mínima para $\mathrm{k}=1$, isto é, para parcelas formadas de uma única planta. Para $\hat{\rho}<0$, essa variância é função decrescente de $k$, ou seja:

$\mathrm{f}(\mathrm{k})=\mathrm{V}(\hat{\mathrm{m}})=\left(\frac{\sigma^{2}}{\mathrm{~N}}\right)[1+(\mathrm{k}-1) \hat{\rho}]$.

Havendo bordadura entre as parcelas de k plantas úteis, em $\mathrm{n}$ fileiras, sendo $\mathrm{N}$ o número total de plantas por tratamento, $\mathrm{N}=\mathrm{Kr}$ ( $\mathrm{N}$ constante) e $\mathrm{r}$ o número de repetições, o número total de plantas por parcela, $\mathrm{K}$, será então:

$K=(n+2 b)\left(\frac{k}{n}+2 b\right)=\left(1+\frac{2 b}{n}\right)(k+2 b n), n>1$.

Como $\mathrm{N}$ é o número total de plantas por tratamento e r o número de repetições:

$\mathrm{N}=\mathrm{Kr}=\left(1+\frac{2 \mathrm{~b}}{\mathrm{n}}\right)(\mathrm{k}+2 \mathrm{nb}) \mathrm{r}$,

e a variância da média do tratamento será então:

$\mathrm{V}(\hat{\mathrm{m}})=\frac{\sigma^{2}}{\mathrm{Kr}}=\left(1+\frac{2 \mathrm{~b}}{\mathrm{n}}\right)(\mathrm{k}+2 \mathrm{bn}) \mathrm{r}$, isto é:

$\mathrm{V}(\hat{\mathrm{m}})=\frac{\sigma^{2}}{\mathrm{~N}}\left(1+\frac{2 \mathrm{~b}}{\mathrm{n}}\right)\left(1+\frac{2 \mathrm{bn}}{\mathrm{k}}\right)[1+(\mathrm{k}-1) \hat{\rho}]$,

$\mathrm{k}>1$ e $\hat{\rho}>0$,

onde $b$ é $o$ tipo de bordadura $(b=0,5, b=1, b=2)$, conforme se usar meia bordadura, bordadura completa ou simples, ou bordadura dupla, e n é o número de fileiras de plantio que constitui a área útil da parcela.

$\mathrm{O}$ mínimo dessa função, para $0<\hat{\rho}<1$, será obtido igualando-se a zero as derivadas parciais $\partial \mathrm{V} / \mathrm{dk}$ e $\partial \mathrm{V} / \mathrm{dn}$, respectivamente, para o número $\mathrm{k}$, de plantas úteis e o número $\mathrm{n}$, de fileiras, da área útil da parcela. Portanto, calculando-se as derivadas, igualando-as a zero e resolvendo-se as equações:

$\mathrm{k}^{2} \hat{\rho}-2 \mathrm{bn}(1-\hat{\rho})=0 \mathrm{e}$

$-\mathrm{k}+\mathrm{n}^{2}=0$, obtêm-se:

$\mathrm{n}=\sqrt[3]{\frac{2 \mathrm{~b}(1-\hat{\rho})}{\hat{\rho}}} \hat{\rho}>0 \mathrm{e}$

$\mathrm{k}=\sqrt{\frac{2 \mathrm{bn}(1-\hat{\rho})}{\hat{\rho}}}, \hat{\rho}>0$, pois $\mathrm{k}=\mathrm{n}^{2}$,

ou seja, $\mathrm{f}(\mathrm{n}, \mathrm{k})$ são as coordenadas do ponto mínimo da variância $\mathrm{V}(\hat{\mathrm{m}})$, isto é:

$\mathrm{f}(\mathrm{n}, \mathrm{k})=\mathrm{V}(\hat{\mathrm{m}})=\frac{\sigma^{2}}{\mathrm{~N}}\left(1+\frac{2 \mathrm{~b}}{\mathrm{n}}\right)\left(1+\frac{2 \mathrm{bn}}{\mathrm{k}}\right)[1+(\mathrm{k}-1) \hat{\mathrm{p}}]$.
Como a área dos experimentos de campo com plantas perenes arbóreas é uma função de $\mathrm{N}=\mathrm{Kr}$, considere-se dois experimentos com parcelas de, respectivamente, $\mathrm{k} \mathrm{e}$ k' plantas úteis, em n e n' fileiras. As variâncias correspondentes serão:

$V(\hat{m})=\frac{\sigma^{2}}{N}\left(1+\frac{2 b}{n}\right)\left(1+\frac{2 b n}{k}\right)[1+(k-1) \hat{p}], k>1$ e $\hat{\rho}>0$,

$\mathrm{V}^{\prime}(\hat{\mathrm{m}})=\frac{\sigma^{2}}{\mathrm{~N}^{\prime}}\left(1+\frac{2 \mathrm{~b}^{\prime}}{\mathrm{n}^{\prime}}\right)\left(1+\frac{2 \mathrm{~b}^{\prime} \mathrm{n}^{\prime}}{\mathrm{k}^{\prime}}\right)\left[1+\left(\mathrm{k}^{\prime}-1\right) \hat{\rho}\right], \mathrm{k}^{\prime}>1 \mathrm{e}$ $\hat{\rho}>0$, onde,

$\mathrm{N}=\mathrm{rK}, \mathrm{N}^{\prime}=\mathrm{r}^{\prime} \mathrm{K}$ ', sendo $\mathrm{K}$ e $\mathrm{K}^{\prime}$ os números totais de plantas por parcela, e, $\mathrm{N}$ e N' os números totais de plantas de cada tratamento nos experimentos. Dessa forma tem-se:

$$
\frac{N^{\prime}}{N}=\frac{\left(1+\frac{2 b^{\prime}}{n^{\prime}}\right)\left(1+\frac{2 b^{\prime} n^{\prime}}{k^{\prime}}\right)\left[1+\left(k^{\prime}-1\right) \hat{\rho}\right]}{\left(1+\frac{2 b}{n}\right)\left(1+\frac{2 b n}{k}\right)[1+(k-1) \hat{\rho}]} .
$$

Como as áreas de cada um dos experimentos são proporcionais aos números totais de plantas, tem-se também:

$$
\frac{A^{\prime}}{A}=\frac{\left(1+\frac{2 b^{\prime}}{n^{\prime}}\right)\left(1+\frac{2 b^{\prime} n^{\prime}}{k^{\prime}}\right)\left[1+\left(k^{\prime}-1\right) \hat{\rho}\right]}{\left(1+\frac{2 b}{n}\right)\left(1+\frac{2 b n}{k}\right)[1+(k-1) \hat{\rho}]} .
$$

Sendo, respectivamente, r e r' o número de repetições para cada experimento, tem-se que:

$\frac{\mathrm{A}^{\prime}}{\mathrm{A}}=\frac{\mathrm{N}^{\prime}}{\mathrm{N}}=\frac{\mathrm{r}^{\prime} \mathrm{K}^{\prime}}{\mathrm{rK}}$, ou seja:

$\frac{A^{\prime}}{A}=\frac{r^{\prime} K^{\prime}}{r K}$, ou ainda:

$\frac{\mathrm{r}^{\prime}}{\mathrm{r}}=\frac{\mathrm{K}}{\mathrm{K}^{\prime}} \times \frac{\mathrm{A}^{\prime}}{\mathrm{A}}$.

\section{Resultados e Discussão}

Como $f(k)=V(\hat{m})=\left(\frac{\sigma^{2}}{N}\right)[1+(k-1) \hat{\rho}]$, considerese, por exemplo, $\mathrm{N}=10$ plantas e $\hat{\rho}= \pm 0,050$. É simples verificar que o mínimo da variância se dá para $\mathrm{k}=1$, independentemente do sinal de $\hat{\rho}$. Se, porém, $\mathrm{k}>1$ e $\hat{\rho}>0$, essa variância é função crescente de $\mathrm{k}$. Mas se $\mathrm{k}>1$ e $\hat{\rho}<0$ ela é função decrescente de $\mathrm{k}$, ou seja: 
tomando $\hat{\rho}=0,050$

Para $\mathrm{k}=1, \mathrm{f}(1)=\left(\sigma^{2} / 10\right)$

Para $\mathrm{k}=2, \mathrm{f}(2)=\left(\sigma^{2} / 10\right) 1,05$

Parak $=5, \mathrm{f}(5)=\left(\sigma^{2} / 10\right) 1,20$

Para $\mathrm{k}=10, \mathrm{f}(10)=\left(\sigma^{2} / 10\right) 1,45$;

tomando $\hat{\rho}=-0,050$

Para $\mathrm{k}=1, \mathrm{f}(1)=\left(\sigma^{2} / 10\right)$

Parak $=2, \mathrm{f}(2)=\left(\sigma^{2} / 10\right) 0,95$

Para $\mathrm{k}=5, \mathrm{f}(5)=\left(\sigma^{2} / 10\right) 0,80$

Para $k=10, f(10)=\left(\sigma^{2} / 10\right) 0,55$.

De modo geral, o mínimo se daria então para $\mathrm{k}=\mathrm{N}$ ou $\mathrm{r}=1$, mas com duas restrições:

a) deve-se ter $1+(\mathrm{k}-1) \hat{\rho} \geq 0$;

b) deve haver um número considerável de repetições $r \geq 2$, que permita obter um número razoável de graus de liberdade para o resíduo, pelo menos dez, para que se tenha bons resultados.

Rossetti et al. (1991, 1996) mostraram que o coeficiente de correlação intraclasse $\hat{\rho}$, em experimentos de campo com cajueiro, varia entre 0,0885 e 0,1361 .

Tomando-se, nesse intervalo $\hat{\rho}=0,0983$, por exemplo, num experimento com bordadura simples ou completa $(b=1)$ entre as parcelas, é possível passar-se de um experimento com $\mathrm{k}=20$ plantas úteis em $\mathrm{n}=4$ fileiras, para outro com k' $=6$ plantas úteis em n' $=2$ fileiras, sem mudar a variância da média de cada tratamento, cuja relação entre as áreas será:

$$
\frac{\mathrm{A}^{\prime}}{\mathrm{A}}=\frac{4,97167}{6,02217}=0,82=82 \% \text {. Isto é }: \mathrm{A}^{\prime}=82 \% \text { de } \mathrm{A} \text {. }
$$

O segundo experimento, de área (A') ocuparia $82 \%$ da área (A), do primeiro. Há, portanto, uma economia de $18 \%$ da área, sem perda de precisão para o experimento.

No caso vertente, os número $\mathrm{K}$ e $\mathrm{K}$ ' de plantas por parcela serão, respectivamente:

$$
\begin{aligned}
& K^{\prime}=(n+2)\left(\frac{k}{n}+2\right)=(4+2)\left(\frac{20}{4}+2\right), \log 0 \mathrm{~K}=42 \\
& K^{\prime}=(2+2)\left(\frac{6}{2}+2\right), \log 0 K^{\prime}=20
\end{aligned}
$$

Portanto:

$$
\frac{\mathrm{r}^{\prime}}{\mathrm{r}}=\frac{\mathrm{K}}{\mathrm{K}^{\prime}} \times \frac{\mathrm{A}^{\prime}}{\mathrm{A}}=\frac{42}{20} \times 0,82=1,722 .
$$

Logo r' $=1,722$ r. Se o experimento com $k=20$ plantas úteis tiver cinco repetições $(r=5)$, o experimento com k' $=6$ plantas úteis por parcela deverá ter $r^{\prime}=1,722$ x $5=8,61$, isto é, oito ou nove repetições.

Note-se que mesmo aumentando-se o número de repetições, de $r=5$ para r' $=8$ ou 9 , o total de plantas por tratamento será reduzido de $\mathrm{N}=5 \times 42=210$ para $\mathrm{N}^{\prime}=8 \times 20=160$ ou N' $=9 \times 20=180$, com redução efetiva de $23,8 \%$ ou $14,3 \%$ no número de plantas necessárias, para o novo experimento, conforme se utilize oito ou nove repetições.

Nas pesquisas com seringueira, por exemplo, onde, segundo Rossetti \& Pimentel-Gomes (1987), $0,0647<\hat{\rho} \leq 0,2967$, num experimento em blocos ao acaso com $r=3$ repetições, com bordadura simples $(\mathrm{b}=1)$ entre as parcelas de $\mathrm{k}=16$ plantas úteis em $\mathrm{n}=4$ fileiras, portanto com um total de $\mathrm{K}=36$ plantas em cada parcela, detectou-se grande variabilidade dentro de cada tratamento, o que tem dificultado a detecção de diferenças significativas entre eles. Se num experimento nessas condições, em que se obteve $\hat{\rho}=0,0789$, se usassem parcelas de $\mathrm{k}^{\prime}=4$ plantas úteis em n' $=2$ fileiras, obter-se-ia r' $=6,8$, podendose utilizar seis ou sete repetições. Utilizando-se r' $=7$ repetições, ter-se-ia, rigorosamente na mesma área, um experimento com parcelas de $\mathrm{K}^{\prime}=16$ plantas ao todo, $61 \%$ menores ou com menos plantas do que as do experimento original, por conseguinte, com muito maior precisão do que o primeiro, uma vez que o erro experimental seria estimado mais fidedignamente, $o$ que daria maior precisão aos testes aplicados e mais confiabilidade aos resultados obtidos.

Com o aumento do número de repetições usando parcelas pequenas, há diminuição da área do experimento, do número de plantas necessárias, portanto, dos custos operacionais. Além disso, obtém-se maior precisão experimental.

Na prática, entretanto, há, às vezes, restrições que impedem o uso do número desejável de repetições, sendo a mais comum relativa à área total do experimento. Assim sendo, a alternativa sugerida neste trabalho, para aumentar o número de repetições e a eficiência dos experimentos, reduzindo, ao mesmo tempo, a quantidade de plantas necessárias e a área experimental, permite maior precisão à pesquisa agronômica de campo, com plantas perenes arbóreas, com custos mais reduzidos. 


\section{Conclusões}

1. Em experimentos de campo com plantas perenes arbóreas, a diminuição da variância da média de cada tratamento é obtida pelo uso de parcelas pequenas.

2. O uso de maior número de repetições com parcelas pequenas possibilita diminuir o tamanho da área experimental e o número de plantas necessárias no experimento, reduzindo os custos da pesquisa.

3. O uso de maior número de repetições com parcelas pequenas permite aumentar a precisão do experimento como um todo, das estimativas do erro experimental, dos parâmetros e dos efeitos de tratamentos, propiciando maior confiabilidade aos resultados da pesquisa.

\section{Referências}

BANZATTO, D. A.; KRONKA, S. do N. Experimentação agrícola. 3. ed. Jaboticabal: Funep/ Unesp, 1995. $247 \mathrm{p}$.

DAGNELIE, P. Théorie et méthodes statistiques. Paris: Agronomiques de Gembloux, 1977. v. 2, 248 p.

GENEZI, A.; LAHAV, E.; PUTTER, J. Determination of optimal plot size in banana experiments. Fruits, Paris, v. 35 , n. 1 , p. $25-28,1980$.

McRAE, K. B.; RYAN, D. A. J. Design and planning of long-term experiments. Canadian Journal of Plant Science, Ottawa, v. 76, n. 4, p. 595-602, 1996.

ORTIZ, R. Plot techniques for assessment of bunch weight in banana trials under two systems of crop management. Agronomy Journal, Madison, v. 87, n. 1, p. 63-69, 1995.

PEARCE, S. C. Field experimentation with fruit trees and other perennial plants. 2. ed. Maidstone: East Malling CAB, 1975. 183 p. (Technical Communication, 23).

PEARCE, S. L.; MOORE, C. S. Reduction of experimental error in perennial crops using adjustment by neighboring plots. Experimental Agriculture, Cambridge, Inglaterra, v. 12 , n. 3 , p. 267-272, 1976.

PIMENTEL-GOMES, F. Curso de estatística experimental. 13. ed. Piracicaba: Nobel, 1990. 468 p.

PIMENTEL-GOMES, F. O problema do tamanho das parcelas em experimentos com plantas arbóreas. Pesquisa Agropecuária Brasileira, Brasília, v. 19, n. 12, p. 15071512, dez. 1984.

ROSSETTI, A. G. Planejamento de experimentos de nutrição e adubação com plantas perenes arbóreas. Fortaleza: Embrapa-CNPAT, 1994. 50 p. (Documentos, 13).

ROSSETTI, A. G.; ALMEIDA, J. I. L. de; PARENTE, J. I. G.; BARROS, L. de. M. Tamanho ótimo de parcela para experimentos com cajueiro comum. Revista Brasileira de Fruticultura, Cruz das Almas, v. 13, n. 2, p. 117 122, 1991.

ROSSETTI, A. G.; BARROS, L. de M.; ALMEIDA. J. I. L. de. Tamanho ótimo de parcelas para experimentos de campo com cajueiro anão precoce. Pesquisa Agropecuária Brasileira, Brasília, v. 31, n. 12, p. 843852, dez. 1996.

ROSSETTI, A. G.; PIMENTEL-GOMES, F. A method for the determination of optimum plot size in experiments with rubber tree (Hevea). Journal of Natural Rubber Research, Kuala Lumpur, v. 2, n. 3, p. 135-141, 1987.

ROSSETTI, A. G.; PIMENTEL-GOMES, F. Determinação do tamanho ótimo de parcelas em ensaios agrícolas. Pesquisa Agropecuária Brasileira, Brasília, v. 18, n. 5, p. 477-487, maio 1983.

SMITH, H. F. An empirical law describing heterogeneity in the yield of agricultural crops. Journal of Agricultural Science, Cambridge, Inglaterra, v. 28, p. 1-23, 1938.

SWALLOW, W. H. Statistical approaches to studies involving perennial crops. HortScience, Alexandria, v. 16, n. 5, p. 634-636, 1981. 